\title{
High speed simultaneous single particle impedance and fluorescence analysis on a chip
}

\author{
Hywel Morgan *, David Holmes, Nicolas G. Green \\ School of Electronics and Computer Science, University of Southampton, Highfield, Southampton SO17 1BJ, United Kingdom
}

Available online 22 December 2005

\begin{abstract}
In this paper, we describe the design and function of a microchip which is used to detect, analyse and count single micron-sized particles at high speed. The device uses multi-frequency electrical impedance together with single particle fluorescence spectroscopy. Impedance is measured using microelectrodes fabricated within a microfluidic channel. Optical measurements are made by focussing a beam of light into a detection volume of the order of the size of the particle. Particles flowing through the device are dynamically focussed into the detection volume using dielectrophoresis. The operating principle of the device is demonstrated by detecting and analysing fluorescent latex particles at high speed.
\end{abstract}

(C) 2005 Elsevier B.V. All rights reserved.

PACS: 84.37

Keywords: High-throughput analysis; Microfluidics; Single particle analysis; Biochip

\section{Introduction}

The rapid development of biochip technology has led to demands for new techniques that are capable of non-contact manipulation, analysis and separation of micron-scale particles such as cells and latex beads, with applications in areas such as: cell analysis or bead-based chemical synthesis and analysis. Microfabrication can be used to produce a range of sophisticated microfluidic devices and a number of different designs of biochips capable of counting and analysing particles have been reported [1-4]. The general principle is relatively simple: particles flow through a microfluidic channel in single file and at high speed using externally generated hydrostatic pressure. The particles are focussed into a small detection volume (generally of the order of the size of a particle) where different parameters can be measured and analysed. In this work, microelectrodes were fabricated in a micro-channel to measure single

\footnotetext{
${ }^{*}$ Corresponding author. Tel.: +44 2380 593330; fax: +44 2380593029. E-mail address: hm@ecs.soton.ac.uk (H. Morgan).
}

particle impedance and simultaneously an optical detection system was employed to measure particle fluorescence. Each of the three components: focussing; impedance detection; and optical detection is described in further detail.

\section{Focussing}

For an efficient high-throughput single particle analysis system, it is important that the particles are efficiently focussed, so that they all pass through a small detection region. This is usually accomplished using hydrodynamic focussing [1-3], where two outer fluid streams flow at a higher pressure and compress a central sample stream, which contains the cells or particles. This type of focussing is one dimensional, and produces a vertical sheet of particles rather than a beam. Nevertheless, prototype particle analysis chips have been developed using this method [13]. However, while it is relatively easy to implement hydrodynamic focussing of a fluid in one-dimension, confinement of a sample stream in two-dimensions may be necessary. 
Commercial flow cytometers employ co-axial focussing, but complex fabrication schemes are required to produce true 2-D focussing in planar microfabricated devices.

An alternative to hydrodynamic focussing is electrokinetic focussing, where DC fields are used to focus particles and liquids by a combination of electrophoresis and electroosmosis $[5,6]$. Devices have been made that are capable of detecting and counting particles optically [4-6]. Generally the throughput of these devices is low, with a typical rate of tens of particles/s [5].

Alternating current (AC) fields, rather than DC fields can also be used to move and manipulate particles and fluids in a flow-through system. This technique, called dielectrophoresis (DEP), relies on dipole forces in non-uniform electric fields [7]. The advantage of the method is that the focussing force acts on the particles rather than on the fluid. The focussing of particles using DEP has been described in detail elsewhere [8,9], where two pairs of electrodes are fabricated along the length of a channel, one set on the top and one on the bottom half of the device, as

(a)
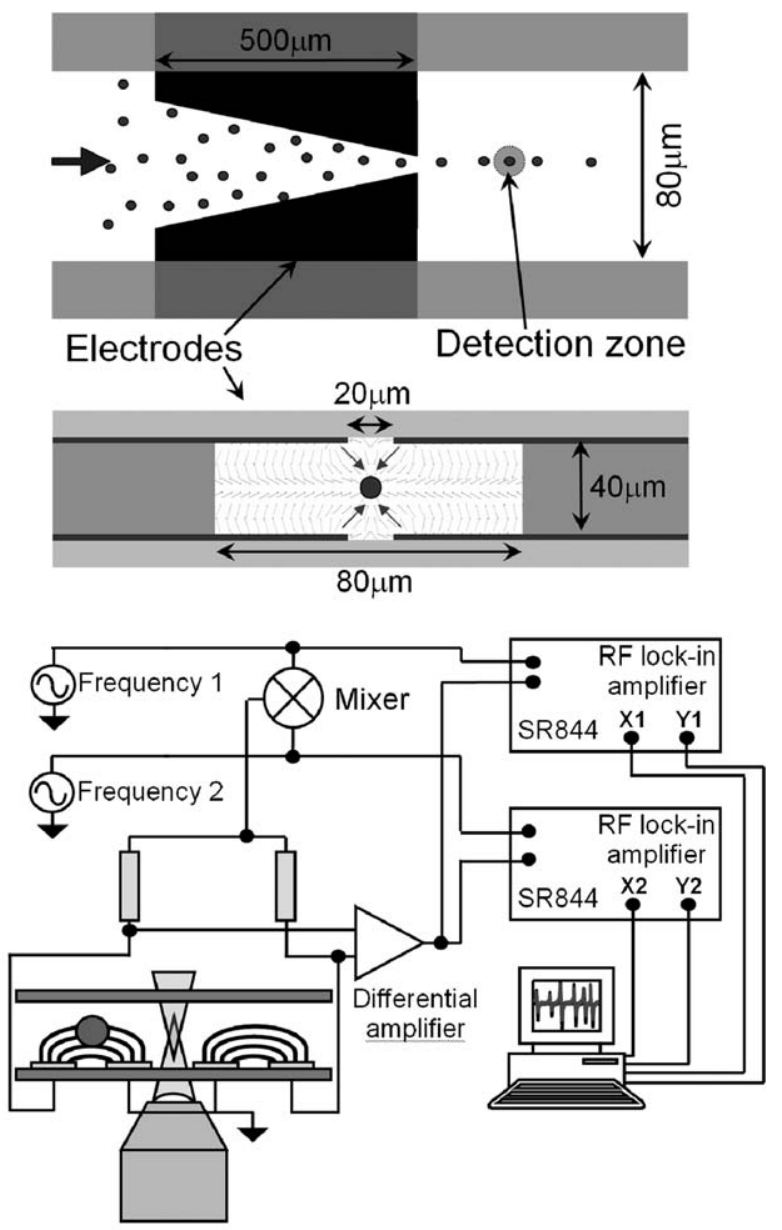

(b)

Fig. 1. (a) Diagram of the electrode layout for 2-D dielectrophoretic focussing of particles in a fluid stream, showing view from above the channel and a cross-section of the channel. (b) Diagram of the system used to measure simultaneous optical and impedance data. Particle impedance is measured at several discrete frequencies using a bridge circuit together with an instrumentation amplifier and signal lock-in. shown schematically in Fig. 1(a). At high field frequencies $(10-20 \mathrm{MHz})$ a negative dielectrophoretic force can be produced, which repels particles from the edges of the electrodes and into a tight beam in the channel centre. The focussing of a range of particles using this method has been demonstrated, down to diameters of $40 \mathrm{~nm}$ [9]. For micron scale particles, the size of the focussing region is the size of a single particle but for nanoparticles, outward diffusion results in a steady state beam diameter of a few microns for $40 \mathrm{~nm}$ particles.

\section{Particle detection}

\subsection{Optical}

Detection of fluorescent particles passing through the microchip was performed using a three colour fluorescence microscope system. The optical system was constructed around an infinity corrected objective lens (40X 0.75 N.A., Nikon), with beams from two lasers (at $532 \mathrm{~nm}$ and $633 \mathrm{~nm}$ ) coupled into the objective. Both beams were focussed to a spot of $5 \mu \mathrm{m}$ diameter at the mid-height of the channel and also mid-point between the microelectrodes used for measuring impedance (see Fig. 1(b)). The fluorescence emission from the particles was captured by the objective lens and passed through dichroic filter sets (Chroma), band pass filters (Chroma) and detected using photomultipliers (Hamamatsu). The fluorescence emission was spatially filtered using a pinhole in front of each photomultiplier, to give a detection volume of the order of $10 \mathrm{fL}$. Using this system, fluorescence could be detected in the following three windows: $550-600 \mathrm{~nm}$ (corresponding to Сy3); 650-690 nm (Су5) and >700 nm (Су7).

\subsection{AC impedance}

Dielectric spectroscopy of particles is a well known technique $[10,11]$ which can be used to non-invasively measure several cellular parameters such as size, membrane capacitance, cytoplasmic conductivity, and for the case of latex particles, the surface charge density, which in turn is related to surface chemistry. Fig. 1(b) shows a schematic diagram of the system which can measure several simultaneous discrete frequencies, typically between $10 \mathrm{kHz}$ and $5 \mathrm{MHz}$, using differential amplifiers. A similar system has been presented previously [12], however in our system the electrodes are fabricated only on the bottom of the channel, making fabrication and alignment easier at the expense of a reduction in sensitivity. The source signal frequencies are digitally synthesised, mixed and then applied to the electrodes through two bridge balance resistors giving typical electrode voltages of between 200 and $300 \mathrm{mV}$. In order to ensure sufficient sensitivity, the electrodes and channel dimensions were of the order of the size of particle to be measured, e.g. for blood cells (5-10 $\mu$ m diameter) the channel had a height and width of $20 \mu \mathrm{m}$, with electrodes of gap and width $10 \mu \mathrm{m}$. Differential voltages were mea- 

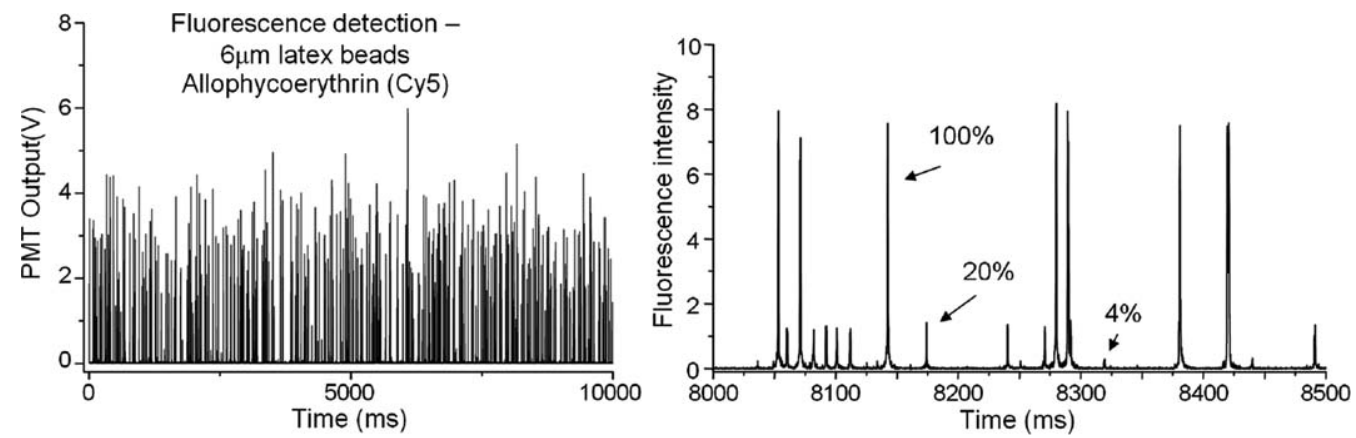

Fig. 2. Photomultiplier signal for beads loaded with different amounts of fluorescent dye, showing a long period of data on the left and a close-up of the signal on the right.
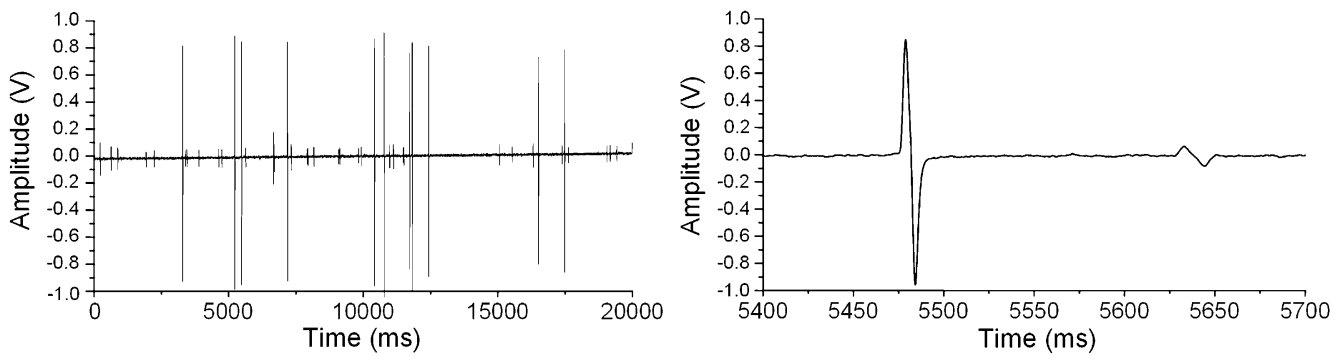

Fig. 3. Time trace of an impedance spectrum for two different sized latex beads showing a long period of data on the left and a close-up of the signal on the right.

sured across each arm of the bridge using a high speed instrumentation amplifier (Analog Devices) and lock-in amplifier (Stanford Research), one for each probe frequency. This circuit arrangement produces a characteristic complex voltage signal which has two peaks, each one corresponding to the movement of a particle through one half of the two sets of electrode pairs. For both optical and impedance collection, the data was digitised using a 16 bit A/D card (National Instruments), time-referenced and stored on hard disc for post-processing using N.I. LabVIEW software.

\section{Results and discussion}

In order to characterise the optical detection system, $6 \mu \mathrm{m}$ diameter fluorescently loaded latex particles with allophycocyanine (Cy5) (Molecular Probes) were used. Particles were obtained with a range of different (but well defined) relative fluorescent intensities. Fig. 2 shows the output signal from the Cy5 photomultiplier channel as a function of time. The three distinct fluorescent intensities of the particles can be clearly resolved at better than $5 \mathrm{~ms}$ time intervals.

An impedance spectrum obtained from single particles passing through the device is shown in Fig. 3. The sample was a mixture of 2 and $4.5 \mu \mathrm{m}$ diameter latex particles suspended in $100 \mathrm{mM}$ phosphate buffered saline (PBS), $\mathrm{pH}$ 7.0, an electrolyte which is representative of physiological saline. The figure shows the $X$-component of the impedance (real signal) measured at $300 \mathrm{kHz}$. There is a
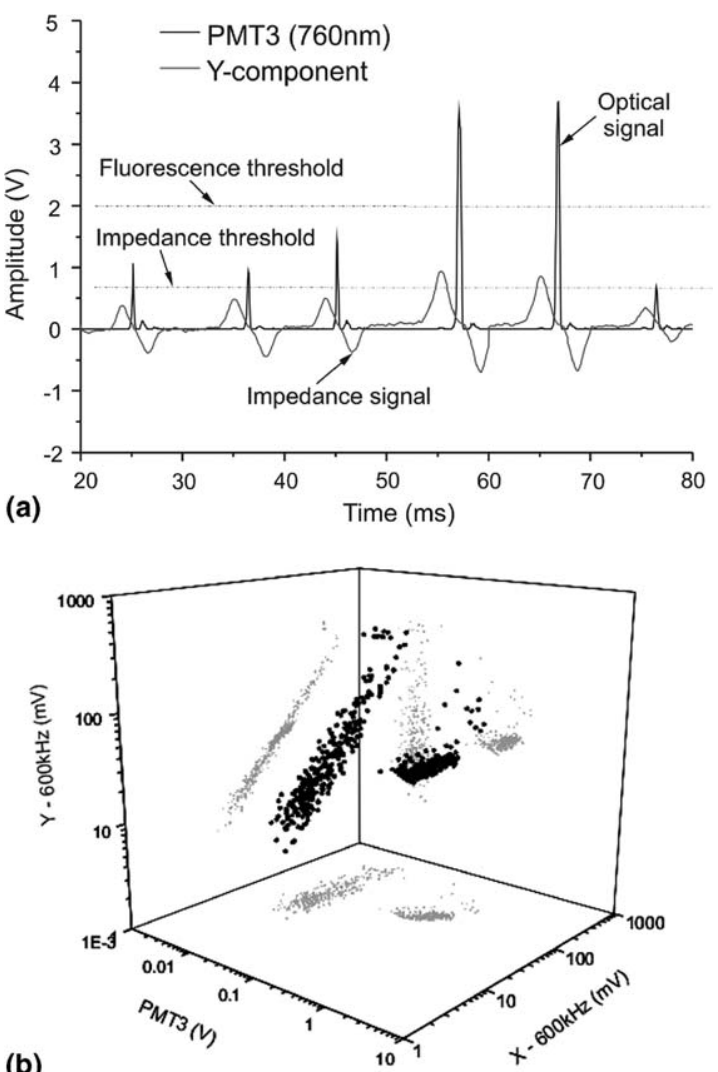

Fig. 4. (a) Time trace of a combined impedance and fluorescence signal for different latex beads. (b) Scatter plot showing individual data points for latex particles as a function of impedance (at $600 \mathrm{kHz}$ ) and fluorescence. 
double peak in the impedance spectrum due to the transit of particles across both pairs of electrodes: a positive signal occurs when the particle crosses the first set of electrodes; a negative signal in crossing the second set. The time difference between the two peaks gives the velocity of the particle. At this frequency the impedance amplitude is dominated by the size of the particle: the impedance signal from the smaller latex particle has a lower magnitude than that from the larger.

Fig. 4(a) shows a combined impedance and optical spectrum for two different sizes of latex particles $(4.5$ and $5.5 \mu \mathrm{m}$ diameter), loaded with fluorescent dye. The figure clearly shows a close correlation between the larger particles, with the greater fluorescence output, and the smaller particles which have lower fluorescence emission intensity. Fig. 4(b) gives an example of a scatter plot for two different sets of latex particles showing the difference in impedance and fluorescence properties. Both particles are of similar size ( 4.5 and $5.5 \mu \mathrm{m}$ diameter), but one population has a large fluorescence intensity and gives a significant photomultiplier (PMT) signal (note log scale along this axis). The $y$ - and $z$-axis show the corresponding impedance data for these particles, the real and imaginary value measured at $600 \mathrm{kHz}$. There is a clear difference in the populations of these two particle types, with the larger particles giving a tighter distribution in intensities.

\section{Conclusion}

A microfabricated device has been developed and fabricated for simultaneous optical and electrical impedance analysis of single particles in a high-throughput system. Results show that it is possible to discriminate between different sizes and types of particles based on both fluorescent intensity and impedance signal at different frequencies.

\section{Acknowledgement}

This work was funded by EPSRC under project number GR/R28942.

\section{References}

[1] J. Krüger, K. Singh, A. O’Neill, C. Jackson, A. Morrison, P.J. O'Brien, Micromech. Microeng. 12 (2002) 486.

[2] N. Pamme, R. Koyama, A. Manz, Lab Chip 3 (2003) 187.

[3] A. Wolff, I.R. Perch-Nielsen, U.D. Larsen, P. Friis, G. Goranovic, C.R. Poulsen, J.P. Kutter, P. Telleman, Lab Chip 3 (2003) 22.

[4] A.Y. Fu, C. Spence, A. Scherer, F.H. Arnold, S.R. Quake, Nat. Biotech. 17 (1999) 1109.

[5] D.P. Schrum, C.T. Culbertson, S.C. Jacobson, J.M. Ramsey, Anal. Chem. 71 (1999) 4173.

[6] S.C. Jacobson, J.M. Ramsey, Anal. Chem. 69 (1997) 3212.

[7] H. Morgan, N.G. Green, AC Electrokinetics: Colloids and Nanoparticles, Research Studies Press, Baldock, UK, 2003.

[8] M. Dürr, J. Kentsch, T. Müller, T. Schnelle, M. Stelzle, Electrophoresis 24 (2003) 722.

[9] H. Morgan, D. Holmes, N.G. Green, IEE Proc. Nanobiotechnol. 150 (2003) 76.

[10] E.H. Grant, R.J. Sheppard, G.P. South, Dielectric Behaviour of Molecules in Solution, Clarendon Press, Oxford, 1978.

[11] K.R. Foster, H.P Schwan, Dielectric properties of tissue, in: C.P. Postow (Ed.), CRC Handbook of Biological Effect Electromagnetic Fields, 1995, p. 618.

[12] S. Gawad, L. Schild, P. Renaud, Lab Chip 1 (2001) 76. 